\title{
245 THE ROLE OF FOLK GAMES IN FITNESS DEVELOPMENT
}

Ashok Kumar Nayak, ${ }^{1}$ Pranakrishna Rout ${ }^{2}$ 'Government College of Physical Education, Sambalpur, Odisha, India; ${ }^{2}$ National Institute of Technology, Rourkela, Odisha, India

\subsection{6/bjsm.2010.078725.245}

Youth who lacked in physical courage, fitness and skill were a danger to the community. It is a known fact that the present youth is very sportive. It is his general tendency to participate in different games and sports, which give him maximum pleasure, spontaneity and recognition. Apart from modern games and sports, the folk games also play an unique role in social 
reconstruction, prosperity for development of culture of the society and self. Folk games like Baguddi, Hu tu tu, Bohuchori, Gambhadian (folk games of Odisha) develop better physical fitness and can be compared with modern physical activities. This study was conducted on 50 rural male youths of Nayagarh district of Orissa State; all subjects were selected on random basis. The age of these subjects ranged between 18 and 22 years. The subjects were divided into two groups (experimental and control group). Twelve week training on Gambhandian (a folk activity conducted during Rakshya Bhandan in Orissa) was administered to the entire group with 30 min duration in the morning and evening 6 days a week. The pre- and post-test scores were taken and compared with Sargent's vertical jump. To find out the effect of the training programme the data was treated by applying analysis of co-variance and level of confidence was chosen at 0.05 . Result of the study revealed that the experimental group on Gambhadian training programme can develop explosive power to increase jumping ability of an athlete. 